\title{
Estudo do ensaio de tração do aço LN-28 por meio da Modelagem Matemática: uma experiência com alunos do Curso Técnico em Fabricação Mecânica
}

\section{Study of the traction test of LN-28 steel through Mathematical Modeling: an experience with students of the Technical Course in Mechanical Fabrication}

\author{
João Cândido Moraes Neves \\ Instituto Federal de Educação, Ciência e Tecnologia do Rio Grande do Sul (IFRS) \\ Campus Caxias do Sul, Caxias do Sul, RS, Brasil \\ http://orcid.org/0000-0001-9083-5447, joao.neves@caxias.ifrs.edu.br
}

\begin{abstract}
Anselmo Antunes Turiel
Instituto Federal de Educação, Ciência e Tecnologia de São Paulo (IFSP), São Paulo, SP, Brasil http://orcid.org/0000-0003-0351-5803, anselmoifsp@gmail.com
\end{abstract}

Juliano Cantarelli Toniolo
Instituto Federal de Educação, Ciência e Tecnologia do Rio Grande do Sul (IFRS)
Campus Caxias do Sul, Caxias do Sul, RS, Brasil
http://orcid.org/0000-0002-8850-6481, juliano.toniolo@caxias.ifrs.edu.br

Greice da Silva Lorenzzetti Andreis Instituto Federal de Educação, Ciência e Tecnologia do Rio Grande do Sul (IFRS) Campus Caxias do Sul, Caxias do Sul, RS, Brasil http://orcid.org/0000-0002-8674-0223, greice.andreis@caxias.ifrs.edu.br

\begin{tabular}{l}
\hline Informações do Artigo \\
\hline cc) (†) 8 : \\
\hline
\end{tabular}

Histórico do Artigo

Submissão: 15 de março de 2019.

Aceite: 8 de junho de 2019 .

\section{Palavras-chave}

Modelagem Matemática

Interdisciplinaridade

Contextualização

Ensaio de Tração

\section{Resumo}

Este artigo apresenta parte dos resultados de um projeto de pesquisa desenvolvido no Instituto de Educação, Ciência e Tecnologia do Rio Grande do Sul, Campus Caxias do Sul, sobre a utilização da Modelagem Matemática como estratégia de ensino e aprendizagem em cursos de nível técnico e de tecnologia. O objetivo da pesquisa consistiu em proporcionar um ambiente de ensino e aprendizagem de Matemática de forma contextualizada com outras áreas do conhecimento, utilizando a Modelagem Matemática para a resolução/entendimento de problemas de disciplinas dos cursos técnicos e de tecnologia do Campus. Entende-se que a busca pela conexão entre a Matemática e cursos das áreas técnicas e tecnológicas é essencial para a qualificação do profissional formado por esta instituição. Este artigo, em específico, tem por objetivo apresentar o modelo matemático desenvolvido por alunos do Curso Técnico em Fabricação Mecânica Integrado ao Ensino Médio, para descrever o ensaio de tração utilizando como corpo de prova o aço LN28, usado pela empresa Marcopolo S.A. na produção de componentes para a indústria automotiva. Para a obtenção do modelo matemático utilizou-se um conjunto de dados experimentais coletados na empresa Marcopolo S.A. e o software Scientific Notebook para a geração do modelo. Com o uso da Modelagem Matemática foi possível mostrar aos alunos a aplicabilidade da Matemática no entendimento de problemas relacionados a sua atuação profissional.

\section{Keywords}

Mathematical Modeling

Interdisciplinarity

Contextualization

Traction Test

\section{Abstract}

This article presents part of the results of a research project developed at the Instituto de Educação, Ciência e Tecnologia do Rio Grande do Sul, Campus Caxias do Sul, about the use of Mathematical Modeling as a teaching and learning strategy in technical and technology courses. The 
objective of the research was to provide an environment of teaching and learning of Mathematics in a contextualized way with other areas of knowledge, using Mathematical Modeling to solve/understand problems of the technical and technological courses of the Campus. It is understood that the search for the connection between Mathematics and courses in the technical and technological areas is essential for the qualification of the professional formed by this institution. This article aims to present the mathematical model developed by students of the Curso Técnico em Fabricação Mecânica Integrado ao Ensino Médio, to describe the tensile test using as test body the LN-28 steel, used by the company Marcopolo S.A. in the production of components for the automotive industry. To obtain the mathematical model was used a set of experimental data collected et the company Marcopolo A.S. and the software Scientific Notebook. With the use of Mathematical Modeling it was possible to show students the applicability of Mathematics in understanding problems related to their professional performance.

\section{Introdução}

Este trabalho é resultado de um projeto de pesquisa desenvolvido no ano de 2012 com turmas de nível técnico e de tecnologia do Instituto Federal de Educação, Ciência e Tecnologia do Rio Grande do Sul, Campus Caxias do Sul. Apresenta-se aqui um recorte da pesquisa, tratando dos resultados obtidos com os estudantes do Curso Técnico em Fabricação Mecânica Integrado ao Ensino Médio da Instituição. Como já mencionado no resumo, o objetivo da pesquisa foi contextualizar a Matemática em cursos técnicos e de tecnologia por meio da Modelagem Matemática, pois a busca pela conexão entre a Matemática e cursos das áreas técnicas e tecnológicas é essencial para a qualificação do profissional formado pelo IFRS, Campus Caxias do Sul.

Entre os anos de 2012 e 2018, os Projetos Pedagógicos dos referidos cursos passaram por algumas atualizações, visando atender a legislações e realizar adequações de forma a contribuir com uma melhor qualificação dos egressos. Analisando os Projetos Pedagógicos atuais, dentre os objetivos do curso de nível médio na área de Fabricação Mecânica, destaca-se a execução e gerenciamento, no setor industrial e de prestação de serviços, de atividades relacionadas à operação, funcionamento e manutenção de máquinas, equipamentos e instalações industriais, visando planejar e executar de maneira eficiente os diferentes processos de fabricação mecânica de diferentes peças (IFRS, 2016).

Com este traçado de perfil de egresso, pode-se observar a necessidade desses profissionais estarem preparados para tratar da resolução de problemas, que certamente aparecerão em suas carreiras profissionais. Neste sentido, uma boa formação matemática, baseada no desenvolvimento do raciocínio dedutivo, pode contribuir na construção das habilidades esperadas para esses futuros profissionais.

De acordo com o material elaborado pelo Comitê de Educação do Instituto ABCD (IABCD, [201-], p. 6), 
O domínio da matemática pode ser decomposto em diferentes habilidades, umas complexas e dependentes de competências linguísticas e cognitivas (domínio de vocabulário específico e compreensão de texto), como é o caso da resolução de problemas, e outras que se desenvolvem muito precocemente e parecem não depender da linguagem, como é o caso do senso numérico.

Quando vamos além da definição da matemática e pensamos no seu processo de aquisição e desenvolvimento, podemos pensar que essas habilidades são regidas por diferentes sistemas especializados do cérebro, mas que esses sistemas trabalham de forma integrada e também requerem o domínio de habilidades cognitivas como atenção, organização e memória operacional.

Assim, trabalhar com metodologias de ensino que desenvolvam a resolução de problemas, o senso numérico, a atenção, a organização e a memória operacional, auxilia profissionais que desejam trabalhar com questões de planejamento e acompanhamento de processos dentro da indústria ou no ramo da prestação de serviços, como no caso de alunos de cursos técnicos e tecnológicos. Seguindo este viés, a Modelagem Matemática, além de promover a integração entre a Matemática e a área técnica, auxilia no desenvolvimento de estratégias para a modelagem e de problemas que fazem parte, em algum nível, da atuação profissional desses indivíduos.

A proposta da aplicação da Modelagem Matemática com uma turma do Ensino Técnico Integrado ao Ensino Médio foi baseada no ensaio de tração utilizando como corpo de prova o aço LN-28. Este material é utilizado na fabricação de carrocerias para ônibus pela empresa Marcopolo S.A., a qual disponibilizou laboratórios de ensaios destrutivos para a realização dos experimentos.

\section{Modelagem Matemática}

A Modelagem Matemática vem sendo desenvolvida de modo indireto desde a Pré-História, conforme aponta Burak (1992), por meio da busca constante do homem em compreender e conhecer o seu ambiente. Além disso, o homem procura, desde os tempos mais remotos, resolver os problemas de sua existência com recursos oferecidos pelo próprio meio em que vive (DIAS, 2007).

Segundo Biembengut (2009, p. 7), o termo "Modelagem Matemática" “[...] como processo para descrever, formular, modelar e resolver uma situação problema de alguma área do conhecimento encontra-se já no início do século $X X$ na literatura de Engenharia e Ciências Econômicas". Além disso, a autora coloca que as discussões acerca do tema, bem como suas aplicações na Educação Matemática, ocorreram principalmente na década de 1960, impulsionando a formação de grupos de pesquisa nesta área. No Brasil, as discussões surgiram no final da década de 1970 e início da década de 1980 com pesquisadores como "Aristides C. Barreto, Ubiratan D' Ambrosio, Rodney C. Bassanezi, João Frederico Mayer, Marineuza Gazzetta e Eduardo Sebastiani” (BIEMBENGUT, 2009, p. 8), que impulsionaram a consolidação da Modelagem Matemática na Educação Matemática.

Bassanezi (2002) aborda a Modelagem Matemática como um método científico de pesquisa e também como uma estratégia de ensino e aprendizagem. Para ele, a Modelagem Matemática é 
a "[...] arte de transformar problemas da realidade em problemas matemáticos e resolvê-los interpretando suas soluções na linguagem do mundo real" (BASSANEZI, 2002, p. 26). Como etapas, o autor coloca a experimentação, a abstração, a formulação, a simplificação, a resolução, a validação e a modificação do modelo, caso necessário. A experimentação envolve atividades laboratoriais nas quais se obtêm os dados; a abstração é o procedimento que leva à obtenção e formulação do modelo matemático; a formulação de hipóteses permite ao pesquisador comparar o modelo com as teorias já existentes; a simplificação do modelo é utilizada pois os problemas reais têm muitas variáveis que influenciam nos fenômenos, muitas vezes complexos; a resolução quando se traduz o modelo matemático da linguagem natural das hipóteses para a linguagem matemática coerente; a validação é o processo de verificação se o modelo matemático representa suficientemente o conjunto de dados; a modificação é a etapa para a realização de eventuais ajustes no modelo, a fim de aprimorá-lo por meio dos dados téricos-experimentais. (BASSANEZI, 2002, p. 26-31).

Biembengut (1997), que foi aluna de Bassanezi, traz a Modelagem Matemática como uma contribuição para o ensino e aprendizagem de Matemática nos cursos de Engenharia, oferecendo ao futuro engenheiro habilidades de discernimento, discussão sobre aspectos da área, proporcionando, desta forma, uma autonomia profissional crítica. Em sua tese, defende o protagonismo do aluno no curso escolhido e faz uma interessante comparação com a obra "O Mundo de Sofia" de Gaarder:

[...] que o ensino parta sempre de temas de interesse ou compatível com a realidade do aluno para chegar na teoria que sustenta esta situação. Vale destacar a obra de GAARDER, J. "O mundo de Sofia" em que o autor, com muito brilho, ensina filosofia a partir de questões da realidade que propõe à "protagonista" do livro para refletir e então a conduz a teoria (BIEMBENGUT, 1997, p. 225).

A autora conclui indicando que deve haver uma orientação aos alunos para uma postura crítica frente ao crescente desenvolvimento da tecnologia, e que a inserção da Modelagem Matemática vem a favorecer neste processo.

Ao tratar a Modelagem Matemática como estratégia de ensino e aprendizagem, Biembengut (1997, p. 110) aponta que no processo de Modelagem “[...] o conteúdo não é dissociado da realidade, conectando o que se aprendeu com o que se executou, estimulando a criatividade (condições essenciais para se ter sucesso na sociedade futura) [...]". Além disso, coloca que esse não é um caminho fácil pois o processo exige pesquisa, criatividade e raciocínio. Barbosa $(2004$, p. 2) aponta cinco razões para a inclusão da Modelagem nos currículos: "motivação, facilitação da aprendizagem, preparação para utilizar a matemática em diferentes áreas, desenvolvimento de habilidades gerais de exploração e compreensão do papel sócio-cultural da matemática”.

Barbosa (2001) considera a Modelagem Matemática como uma oportunidade para os alunos questionarem situações por intermédio da Matemática sem procedimentos fixos e com diversas possibilidades de encaminhamentos. Por apresentar este caráter aberto, o autor coloca que não há 
garantia da presença de um modelo matemático na abordagem dos alunos, visto que isso depende da construção feita por eles. Traz a Modelagem Matemática sob o viés da indagação:

A indagação não se limita à explicitação do problema, mas uma atitude que permeia o processo de resolução. Se tomarmos Modelagem de um ponto de vista sóciocrítico, a indagação ultrapassa a formulação ou compreensão de um problema, integrando os conhecimentos de matemática, de modelagem e reflexivo (BARBOSA, 2001, p. 6).

Com esta colocação do autor, entende-se que a Modelagem Matemática pode ser trabalhada em qualquer nível de ensino, desde os anos iniciais até cursos de nível superior, desde que as relações matemáticas envolvidas estejam adequadas a cada nível de ensino. Além disso, nesta perspectiva, o ambiente de ensino pode ser entendido como um "convite" aos alunos para que estes se envolvam com as atividades. Como a Modelagem, para Barbosa (2001), é pautada na indagação, não se trata de uma simples explicitação de um problema, mas sim uma postura do aluno que acompanha todo o processo de resolução, pois todas as etapas dependem de seu interesse com a problemática estabelecida.

Barbosa (2001), a partir de uma análise a trabalhos nacionais e internacionais, classifica as possibilidade de abordagem da Modelagem Matemática em três casos, nos quais o professor dialoga com os alunos em diferentes níveis: no primeiro caso, o professor apresenta o problema com dados qualitativos e quantitativos, cabendo aos alunos o processo de resolução, sem a necessidade de sair da sala de aula; no segundo caso, o professor traz um problema de outra área para os alunos e estes precisam realizar a coleta de informações necessárias para sua resolução, bem como realizar simplificações no modelo possibilitando a resolução do mesmo; no terceiro caso, os alunos escolhem um tema, formulam o problema e o resolvem, passando por todas as etapas do processo de Modelagem.

Neste contexto, identificando a possibilidade de a Modelagem Matemática contribuir de forma significativa na formação de profissionais das áreas técnicas, apresenta-se aqui uma experiência de contextualização da Matemática no Curso Técnico em Fabricação Mecânica Integrado ao Ensino Médio por meio da Modelagem Matemática.

\section{Metodologia}

Em 2012 foi desenvolvido o projeto de pesquisa "Iniciação à Modelagem Matemática no Ensino Básico, Técnico e Tecnológico" no Instituto Federal de Educação, Ciência e Tecnologia do Rio Grande do Sul, Campus Caxias do Sul. O projeto tratou da utilização da Modelagem Matemática como estratégia de ensino e aprendizagem em cursos de nível técnico (Fabricação Mecânica, Plásticos e Química) e de tecnologia (Tecnologia em Processos Metalúrgicos) da Instituição.

Este artigo traz as atividades desenvolvidas com os alunos do segundo ano do Curso Técnico em Fabricação Mecânica Integrado ao Ensino Médio, que foram convidados a participar da pesquisa durante as aulas regulares de Matemática. A turma contava com 30 alunos no ano de 
2012. Como um dos objetivos deste projeto era trabalhar de forma interdisciplinar, trabalhou-se em parceria com uma disciplina técnica da matriz curricular, a qual envolvia fenômenos mecânicos e sua parte experimental, permitindo, desta forma, desenvolver um modelo matemático com dados experimentais e dados teóricos da literatura. O objetivo do trabalho realizado foi de proporcionar uma atividade interdisciplinar para analisar a participação e o interesse dos alunos em uma tentativa de integração entre a Matemática e uma disciplina técnica, fortalecendo a proposta de Ensino Médio Técnico Integrado.

Para a realização da referida integração, a modelagem matemática foi trabalhada da seguinte forma:

1. Definição do tema: A fim de promover a interdisciplinaridade, os professores de Matemática e de Tecnologia Mecânica da turma, autores deste artigo, escolheram trabalhar com a obtenção de um modelo matemático para o ensaio de tração para o aço LN-28. O ensaio de tração foi trabalhado de forma teórica e experimental com o professor de Tecnologia Mecânica e o modelo matemático foi construído com o professor de Matemática.

2. Coleta de dados: Os dados experimentais foram coletados na empresa Marcopolo S.A., por meio de experimentos realizados pelo professor de Tecnologia Mecânica em conjunto com a turma. Os dados tabulados foram fornecidos pela máquina de tração durante os experimentos.

3. Modelo matemático: A construção do modelo matemático foi realizada com o software Scientific Nootebook por meio de ajustes de curvas.

4. Validação do modelo: A validação foi realizada mediante comparação com os dados experimentais disponíveis, analisando o coeficiente de determinação dos ajustes encontrados e a teoria estudada na disciplina de Tecnologia Mecânica.

$\mathrm{Na}$ sequência descreve-se em que consiste o ensaio de tração e, após, apresenta-se o processo de obtenção do modelo matemático construído pelos alunos.

\section{Ensaio de Tração}

Um dos métodos mais usados nos testes em materiais para a construção de componentes, principalmente no ramo mecânico, é o ensaio de tração, em que os materiais são submetidos a certas variações de cargas, verificando o que eles podem absorver antes da ruptura. Como a indústria usa o aço em muitos de seus componentes, é importante a coleta de dados quantitativos e qualitativos sobre este material, obtidos pelo ensaio de tração, para atestar o grau de seguridade dos componentes construídos com ele.

O ensaio de tração é muito usado na indústria automotiva, pois esta realiza vários testes para analisar o grau de sustentação das peças fabricadas, sob o ponto de vista de proporcionar garantias de fabricação. No caso dos fabricantes de carrocerias para ônibus, exige-se uma gama 
de testes para a aprovação das mesmas, garantindo assim que, em situações normais, os componentes automotivos operem com segurança.

O ensaio de tração consiste na aplicação gradativa de uma força de tração uniaxial nas extremidades de um corpo de prova até sua ruptura. Neste tipo de ensaio mede-se a variação do comprimento como uma função da força aplicada. Quando um corpo de prova é submetido a um ensaio de tração, a máquina de ensaio fornece um gráfico que mostra as relações entre a força aplicada e as deformações ocorridas durante o ciclo, conforme representado de forma genérica na Figura 1. Este gráfico é conhecido como "Diagrama Tensão-Deformação". Pelo Sistema Internacional, a unidade da tensão é o Newton por metro quadrado $\left(\mathrm{N} / \mathrm{m}^{2}\right)$, que é denominada Pascal $(\mathrm{Pa})$, ou o seu múltiplo, o megapascal $(\mathrm{MPa})$. A tensão também pode ser apresentada em libras-força por polegada quadrada, denominada psi $\left(1 \mathrm{MPa}=1 \mathrm{~N} / \mathrm{mm}^{2}=145 \mathrm{psi}, 1 \mathrm{psi}=6,9 \times 10^{-3}\right.$ $M P a)$. A unidade de deformação é o metro por metro $(\mathrm{m} / \mathrm{m})$ e, portanto, adimensional (independente do sistema de unidades) (TEÓFILO, 2017).

A partir do gráfico gerado para cada ensaio é possivel analisar a relação entre a tração e a deformação do corpo de prova. A tensão $(\sigma)$ é equivalente a razão da força $(P)$ pela área da seção (A) em relação a qual a força é aplicada, ou seja (HIBBELER, 2010, p. 71),

$$
\sigma=\frac{P}{A}
$$

Figura 1 - Diagrama Tensão-Deformação convencional.

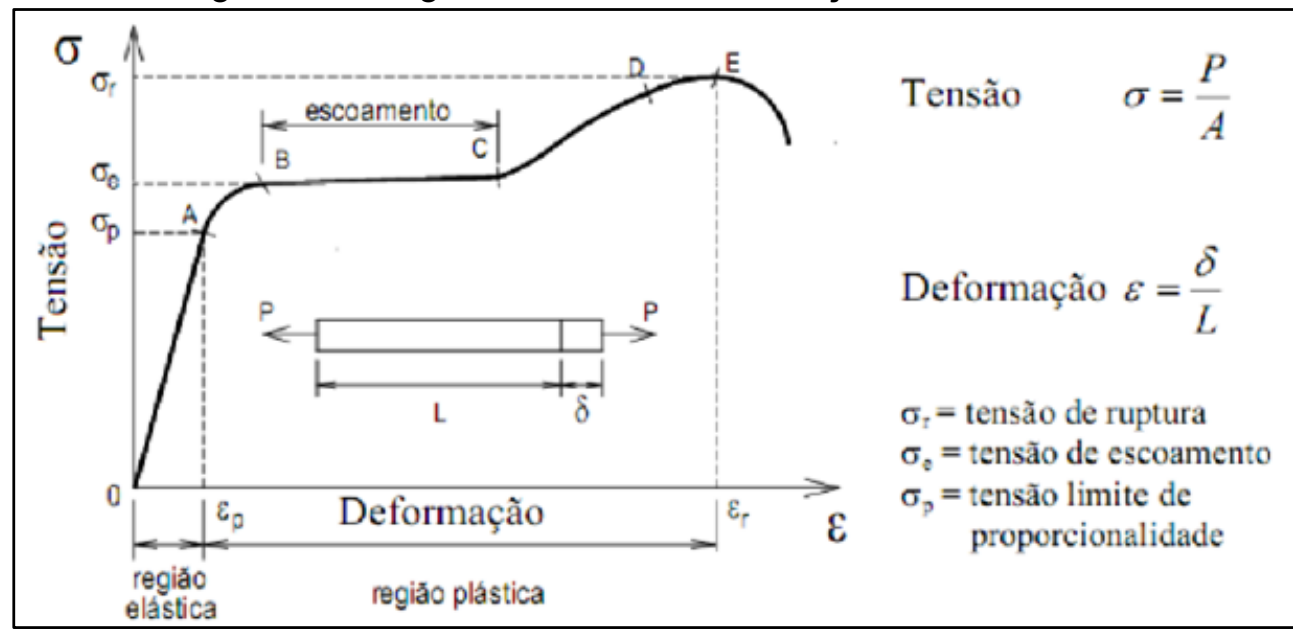

Fonte: Hibbeler (2010, p. 74).

Os elementos apresentados na Figura 1 são utilizados para avaliar diversas propriedades mecânicas dos materiais e para o controle das especificações da matéria-prima fornecida. A região elástica ocorre até quando a tensão limite de proporcionalidade é atingida $\left(\sigma_{p}\right)$. O módulo de elasticidade é a inclinação (coeficiente angular) do segmento linear no gráfico tensão-deformação, e é "uma medida de rigidez ou de resistência do material à deformação elástica, ou seja, quanto maior esse módulo, menor será a deformação elástica resultante da aplicação de uma determinada tensão (maior rigidez)" (TEÓFILO, 2017, p. 144). A deformação plástica consiste em uma 
deformação permanente e não recuperável. Do ponto $A$ ao ponto $B$ observa-se uma curvatura que surge na região plástica, aumentando mais rapidamente com a elevação da tensão, até atingir a tensão de escoamento $\sigma_{e}$. A tensão de ruptura $\sigma_{r}$ corresponde à tensão na qual o material se rompe.

\section{Modelo para o Ensaio de Tração criado pelos alunos}

O estudo de materiais é abordado no Curso Técnico em Fabricação Mecânica Integrado ao Ensino Médio na disciplina de Tecnologia Mecânica. Neste componente curricular são abordados conceitos sobre materiais, tais como: tensão, deformação, propriedades mecânicas dos materiais, carga coaxial (deformação), tração e torção, entre outros. Durante o ensaio de tração, o corpo de prova é submetido a uma certa carga crescente até que haja a ruptura do mesmo. A máquina de ensaio de tração apresenta os valores da deformação do material, bem como o gráfico gerado a partir destes. Este teste tem grande importância para a análise do material a ser usado na fabricação de componentes mecânicos. O aço LN-28 foi escolhido, pois este material é o mais utilizado na fabricação de carrocerias para ônibus, que é o principal produto de fabricação da empresa onde foi realizada a experimentação.

O professor de Tecnologia Mecânica levou a turma para a realização da experimentação no Laboratório de Materiais da empresa Marcopolo S.A.. O equipamento utilizado no ensaio de tração para o aço LN-28 é apresentado na Figura 2.

Figura 2 - Equipamento utilizado no ensaio de tração do aço LN-28.

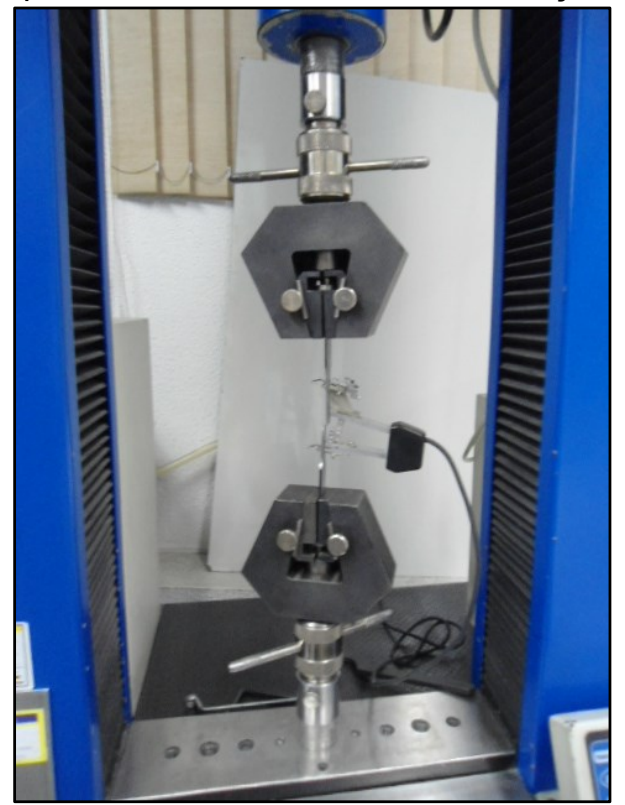

Fonte: Equipamento do Laboratório de Materiais da empresa Marcopolo S.A. (2012).

O experimento consiste em colocar o corpo de prova na máquina e aplicar uma carga axial unilateral variável. Este corpo vai se deformando até sua ruptura. Durante este processo, o corpo de prova apresenta duas fases, uma elástica, onde o corpo de prova volta ao seu estado inicial, quando cessada a ação da carga e, a outra, plástica, onde o corpo não volta mais ao seu estado 
inicial, quando cessada a carga submetida. Quando o corpo de prova atinge a fase plástica e continua submetido à tração, ele rompe. Os dados numéricos apresentados pelo equipamento durante o ensaio de tração foram coletados pelos alunos. Estes dados são apresentados de forma parcial na Tabela 1, uma vez que durante a realização do experimento de tração foram realizadas 878 aquisições de leituras de tempo, deformação e força, até o rompimento do corpo de prova.

Tabela 1 - Dados experimentais coletados no ensaio de tração do aço LN-28.

\begin{tabular}{ccc}
\hline Tempo(s) & Deformação(N/s) & Força(N) \\
\hline 0,016667 & 0 & 67,700 \\
\hline 0,10 & 0,00056563 & 84,625 \\
\hline 0,26667 & 0,00113130 & 98,165 \\
\hline 0,53333 & 0,00056563 & 111,700 \\
\hline 0,85 & 0,00113130 & 125,240 \\
\hline 1,0667 & 0,00113130 & 138,780 \\
\hline 1,25 & 0,00113130 & 152,320 \\
\hline 1,4167 & 0,00113130 & 165,860 \\
\hline 1,5667 & 0,00113130 & 179,400 \\
\hline 1,70 & 0,00169690 & 192,940 \\
\hline 1,7667 & 0,00169690 & 206,480 \\
\hline$\ldots$ & $\ldots$ & $\ldots$ \\
\hline 123,55 & 12,82500000 & 5361,800 \\
\hline 123,57 & 12,84100000 & 3798,000
\end{tabular}

Fonte: Laboratório de Materiais da empresa Marcopolo S.A. (2012).

A partir dos dados experimentais apresentados na Tabela 1, os alunos utilizaram o software Scientific Nootebook nas aulas de Matemática para a elaboração do modelo. No Laboratório de Informática os alunos foram divididos em 5 grupos de 6 alunos cada. Com estes dados os alunos foram tentando vários tipos de ajustes de regressão de curvas: linear, exponencial, logarítmicas, logística e outras, para analisar a que mais se aproximava dos dados experimentais. Todas estas possibilidades foram discutidas com os alunos, explorando as características de cada família de funções.

Por meio do software Scientific Nootebook o melhor ajuste encontrado para os dados apresentados foi o ajuste logarítmico, gerando um modelo matemático para a força de tensão $F(N)$ aplicada ao corpo de prova em função da deformação $x(\mathrm{~mm})$ sofrida pelo mesmo durante o ensaio de tração, dado por:

$$
F(x)=9425,1802-597,71228 \ln (x-0,09585)
$$


$\mathrm{Na}$ Figura 3, apresenta-se a comparação entre os dados experimentais e o modelo matemático obtido. A validação do modelo se deu por meio da análise do coeficiente de determinação. O coeficiente de determinação $\left(R^{2}\right)$ é uma medida de ajustamento de um modelo estatístico linear generalizado em relação aos valores observados. Este coeficiente varia de 0 a 1 , e indica o quanto o modelo consegue explicar o conjunto de dados disponíveis. Quanto maior o seu valor, mais explicativo é o modelo, ou seja, melhor ele se ajusta à amostra (WIKIPÉDIA, 2019). Os alunos chegaram a um valor muito próximo a $1, R^{2}=0,95172$, o que significa que $95,17 \%$ da variável dependente consegue ser explicada pelo modelo. Nestas condições, pode-se afirmar que o modelo obtido apresenta uma boa aproximação com os dados experimentais coletados.

Figura 3 - Comparação entre os dados experimentais (pontos em preto - 878 medições) e o modelo obtido (linha em vermelho) no software Scientific Notebook para o ensaio de tração do aço LN-28.

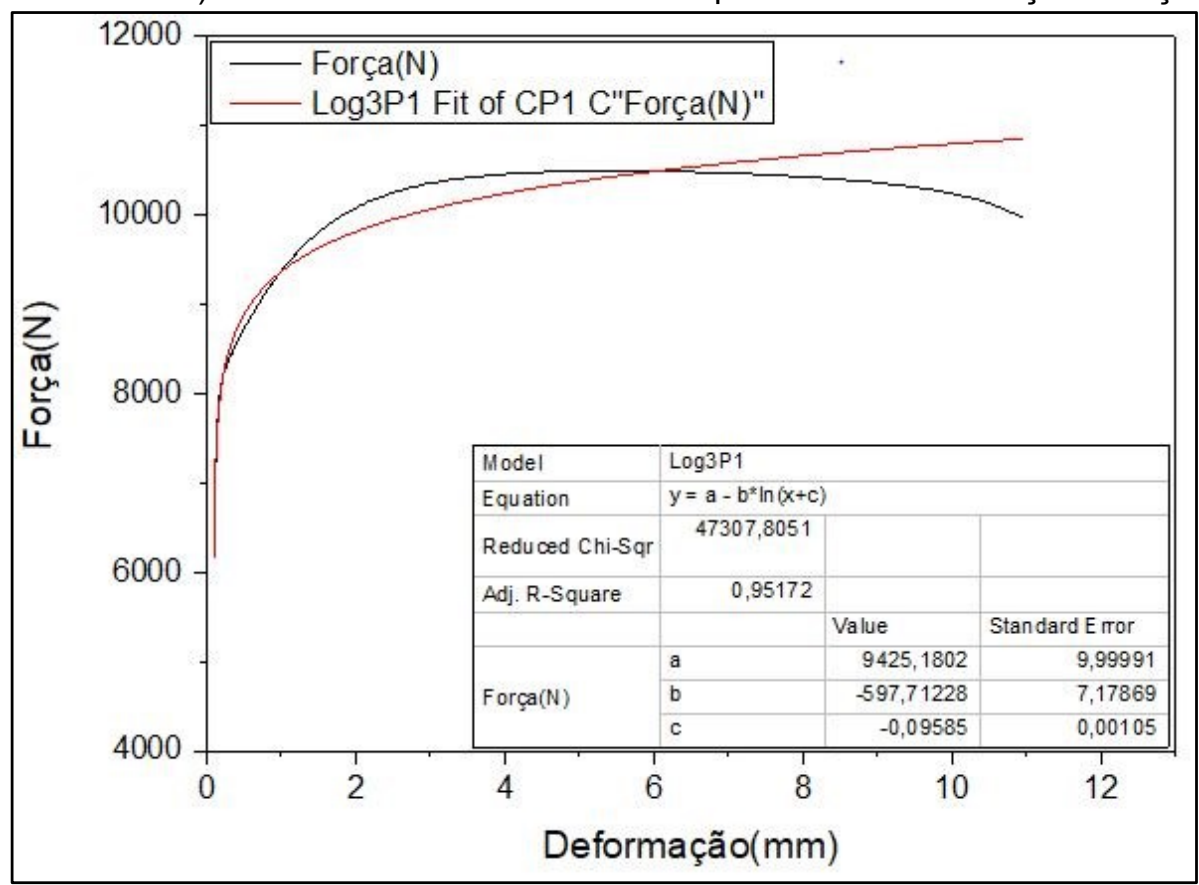

Fonte: Dados da pesquisa (2012).

Vale ressaltar que não houve interesse em trabalhar com o erro neste modelo, pois as ferramentas de análise de erros são abordadas posteriormente no curso na disciplina de Elementos de Máquinas, dedicando este momento à questão interdisciplinar da atividade e à aplicação da Matemática no curso escolhido. Desta forma, este trabalho poderia ser aperfeiçoado posteriormente no curso, quando os alunos tivessem domínio de técnicas, como por exemplo a aferição do ferramental usado.

O gráfico do modelo apresentado na Figura 3 apresenta uma grande discrepância em sua parte final (lado direito). Isto deve-se pela descompressão da máquina após a ruptura do aço LN28, não tendo mais influência sobre o corpo de prova. Este aspecto foi discutido com os alunos, relacionando o domínio matemático do modelo encontrado com a situação real, ou seja, o modelo descreve a amostra até o momento da ruptura do corpo de prova. 
Ao final das atividades desenvolvidas de forma integrada entre as disciplinas de Matemática e Tecnologia Mecânica, foi solicitado aos 30 alunos da turma que respondessem um questionário de forma individual, cujos resultados são apresentados na Tabela 2.

Tabela 2 - Respostas dos alunos referente à aplicação da atividade desenvolvida de forma interdisciplinar.

\begin{tabular}{|l|c|c|c|}
\hline \multicolumn{1}{|c|}{ Questionário } & Sim & Não & Talvez \\
\hline $\begin{array}{l}\text { 1. A atividade de Modelagem Matemática motivou você para o entendimento da } \\
\text { Matemática envolvida no experimento? }\end{array}$ & 29 & 0 & 1 \\
\hline $\begin{array}{l}\text { 2. Foi relevante para o ensino e aprendizagem da Matemática e da Tecnologia } \\
\text { Mecânica usar a Modelagem Matemática como uma metodologia de ensino e } \\
\text { aprendizagem? }\end{array}$ & 28 & 1 & 1 \\
\hline $\begin{array}{l}\text { 3. A interdisciplinaridade foi relevante para mostrar a aplicabilidade da } \\
\text { Matemática em outras áreas do conhecimento? }\end{array}$ & 26 & 3 & 1 \\
\hline $\begin{array}{l}\text { 4. É possível trabalhar as disciplinas de forma integrada, conforme é a } \\
\text { indicação do curso (Ensino Médio Técnico Integrado)? }\end{array}$ & 25 & 1 & 4 \\
\hline $\begin{array}{l}\text { 5. Por meio da Modelagem Matemática é possível promover a integração entre } \\
\text { os alunos para a resolução/entendimento de problemas do cotidiano? }\end{array}$ & 28 & 1 & 1 \\
\hline
\end{tabular}

Fonte: Dados da pesquisa (2012).

Pelas respostas dos alunos ao Questionário foi possível notar uma boa aceitação da atividade, bem como uma compreensão sobre a possibilidade de realmente integrar as disciplinas técnicas às disciplinas propedêuticas do curso.

\section{Considerações finais}

A Modelagem Matemática foi desenvolvida como uma forma de contextualização da Matemática no Curso Técnico em Fabricação Mecânica, isto é, promovendo a interdisciplinaridade que é o foco do Curso Técnico Integrado ao Ensino Médio, em que as áreas técnicas e propedêuticas devem estar correlacionadas. Foi construído um modelo matemático para o ensaio de tração do aço LN-28 com os alunos, desde a etapa de coleta de dados junto à empresa Marcopolo S.A.. O modelo apresentou boa aproximação em relação aos dados experimentais coletados até o momento da ruptura.

Analisando as respostas dos alunos, conforme o questionário proposto no final das atividades desenvolvidas, foi possível constatar a interação entre eles, estimulada pela Modelagem Matemática. Os alunos afirmaram que foi possível ver a aplicação da Matemática no curso e também sua importância para a resolução de problemas do cotidiano. Com o uso da Modelagem Matemática foi possível mostrar aos alunos a aplicabilidade da Matemática no entendimento de problemas relacionados a sua atuação profissional.

Por meio desta pesquisa buscou-se mostrar que a Modelagem Matemática pode entrar de maneira satisfatória em cursos técnicos sem deixar de lado os conteúdos programáticos, 
colaborando no processo de ensino e de aprendizagem da Matemática e na integração com outras áreas do conhecimento.

\section{Referências}

BARBOSA, Jonei Cerqueira. Modelagem Matemática: O que é? Por que? Como? Veritati, Lisboa, Portugal, n. 4, p. 73-80, 2004.

BARBOSA, Jonei Cerqueira. Modelagem na Educação Matemática: contribuições para o debate teórico. In: REUNIÃO ANUAL DA ANPED, 24, 2001. Caxambu. Anais... Caxambu: ANPED, 2001. Disponível em:

$<$ https://www.google.com/url?sa=t\&rct=i\&q=\&esrc=s\&source=web\&cd=3\&cad=rja\&uact=8\&ved=2a hUKEwjvpKbVn4HfAhWIWpAKHQnZBk0QFjACegQIBxAC\&url=http\%3A\%2F\%2F24reuniao.anped .org.br\%2FT1974438136242.doc\&usg=AOvVaw2lq28uN2K5YI05ZBn9f9u4>. Acesso em: 30 ago. 2018.

BASSANEZI, Rodney Carlos. Ensino-Aprendizagem com Modelagem Matemática. São Paulo: Contexto, 2002.

BIEMBENGUT, Maria Salett. 30 Anos de Modelagem Matemática na Educação Brasileira: das propostas primeiras às propostas atuais. ALEXANDRIA: Revista de Educação em Ciência e Tecnologia, v. 2, n. 2, p. 7-32, jul. 2009.

BIEMBENGUT, Maria Salett. Qualidade de Ensino de Matemática na Engenharia: uma proposta metodológica e curricular. 1997. 175 f. Tese (Doutorado em Engenharia de Produção e Sistemas) - Programa de Pós-Graduação em Engenharia de Produção e Sistemas, Universidade Federal de Santa Catarina, Florianópolis, 1997.

BURAK, Dionísio. Modelagem Matemática: ações e interações no proceso de ensinoaprendizagem. 1992. 460f. Tese (Doutorado em Educação) - Programa de Pós-Graduação em Educação. Faculdade de Educação. Universidade Estadual de Campinas, Campinas, SP, 1992.

DIAS, Maria da Graça Andrade. Modelagem no Ensino da Geometria. Graphica, Curitiba, p. 1-9, 2007. Disponível em:

$<$ http://www.exatas.ufpr.br/portal/docs degraf/artigos graphica/MODELAGEM\%20NO\%20ENSIN O\%20DA\%20GEOMETRIA.pdf>. Acesso em: 30 ago. 2018.

HIBBELER, Russel Charles. Resistência dos Materiais. 7. ed. São Paulo: Pearson Prentice Hall. 2010.

$I A B C D$. Instituto $A B C D$. Comitê de Educação. Habilidade matemáticas: o que são, como avaliar e como melhorar. Módulo 6. [201-]. Disponível em: <http://www.institutoabcd.org.br/portal/arquivos/1392132744 modulo6 2701 web.pdf>. Acesso em: 30 ago. 2018.

IFRS. Instituto Federal de Educação, Ciência e Tecnologia do Rio Grande do Sul. Campus Caxias do Sul. Projeto Pedagógico do Curso Técnico em Fabricação Mecânica Integrado ao Ensino Médio. 2016. Disponível em: <https://ifrs.edu.br/caxias/cursos/cursos-tecnicos/fabricacaomecanica-integrado/>. Acesso em: 30 ago. 2018.

TEÓFILO, Jorge. Propriedades dos Materiais: Diagramas tensão-deformação. In.: Estrutura e propriedades dos materiais. 2017. Disponível em: 
<https://jorgeteofilo.files.wordpress.com/2017/01/epm-apostila-capc3adtulo09-propriedades.pdf>. Acesso em: 03 jun. 2019.

WIKIPÉDIA. Coeficiente de determinação. 09 jan. 2019. Disponível em:

$<$ https://pt.wikipedia.org/wiki/Coeficiente de determina\%C3\%A7\%C3\%A3o>. Acesso em: 03 jun. 2019. 